\title{
Un porcentaje de los cánceres de pulmón diagnosticados por rastreo con tomografia computada de baja dosis podrían corresponder a sobrediagnósticos
}

A significant percentage of lung cancers diagnosed by screening with low-dose CT may correspond to overdiagnosis

Ann Intern Med. 2012; 157:776-784

\begin{abstract}
Antecedentes
El rastreo de cáncer de pulmón puede detectar un cáncer que nunca será sintomático (sobre-diagnóstico), conduciendo a sobre-tratamiento. El cambio de tamaño de las lesiones detectadas en el rastreo con tomografía computada de baja dosis (TCBD), expresados como tiempo de duplicación de volumen (TDV), pueden ayudar a distinguir cánceres agresivos de aquellos casos que improbablemente se hagan sintomáticos.
\end{abstract}

\section{Objetivo}

Evaluar el TDV de cánceres de pulmón detectados mediante rastreo con TCBD durante cinco años y su correlación con la evolución clínica como un modo posible de estimar sobrediagnóstico.

\section{Diseño, lugar y pacientes}

Evaluación retrospectiva de un estudio de cohortes, realizado en un único centro (Milán, Italia), en el que se ofreció (entre 2004 y 2005) a voluntarios asintomáticos tabaquistas o ex tabaquistas (de hasta diez años) de al menos 20 pack-year, rastreo de cáncer de pulmón anual y consecutivo durante cinco años con TCBD. Se acordó que no se informaría a los pacientes aquellas lesiones menores o iguales a $5 \mathrm{~mm}$. Para las lesiones mayores el algoritmo de manejo variaba de acuerdo al tipo de lesión encontrada (nueva TCBD, PET-TC, biopsia, cirugía, etc)

\section{Medición de resultados principales:}

EI TDV fue medido mediante TCBD y clasificado como de crecimiento rápido (<400 días), crecimiento lento (entre 400 y 599 días) o indolente ( $\geq 600$ días). La información de la situación vital se valoró en las visitas de seguimiento, mediante llamados telefónicos (en caso de no asistencia) o mediante consulta a registros oficiales municipales.

\section{Resultados}

Se enrolaron y realizaron una TCBD basal 5.203 participantes (66\% varones, $80,3 \%$ fumadores activos). La adherencia a las cinco rondas de TCBD fue del $79,2 \%$. No se pudo obtener información sobre la situación vital de 44 participantes.

Se diagnosticaron 175 casos de cáncer de pulmón durante los cinco años, 55 fueron casos prevalentes ( $1,1 \%$ de la población rastreada, diagnosticados basalmente en la primera TCBD) y 120 de diagnóstico subsecuente (los que interesan al estudio). Del último grupo, 19 casos (15,8\%) fueron diagnósticos nuevos (lesiones no visibles en estudios previos) y de crecimiento rápido (media de VDT de 52 días); 101 (84,2\%) fueron progresivos, incluyendo 70 casos $(58,3 \%)$ de crecimiento rápido, $18(15,0 \%)$ de crecimiento lento y 13 de crecimiento indolente $(10,8 \%)$. La mortalidad específica por cáncer de pulmón fue significativamente $(p=0,01)$ más alta $(9,2 \%)$ por año en pacientes con cánceres nuevos, comparados con los de crecimiento lento o indolente $(0,9 \%$ por año) y no significativamente $(p=0,138)$ más alta al comparar aquellos de crecimiento rápido ( $4,1 \%$ por año) con los de crecimiento lento o indolente $(0,9 \%$ por año).

\section{Conclusiones}

El cáncer de crecimiento lento o indolente comprende aproximadamente $25 \%$ de los casos incidentales, mucho de los cuales podrían haber sido sobrediagnosticados. Para limitar el sobre-tratamiento en estos casos se debería investigar estrategias de resección mínimamente invasiva y tratamientos no quirúrgicos.

Fuente de financiamiento: Asociación Italiana de Investigación de Cáncer.

\section{Comentario}

Si bien este estudio tiene limitaciones metodológicas (análisis retrospectivo, forma de asignar la velocidad de crecimiento de las lesiones nuevas), sus resultados sugieren que hasta un $25 \%$ de los cánceres diagnosticados por la TCBD (los cánceres indolentes y los de crecimiento lento) podrían ser sobre-diagnósticos ${ }^{1-4}$

Estos datos, además de otros no menos significativos como el impacto de la aplicación de una técnica por el daño directo que puede producir, los falsos positivos y los costos que podrían ser detraídos de otros procedimientos más costo-efectivos y con mayor impacto poblacional deben ser tenidos cuidadosamente en cuenta a la hora de recomendar un procedimiento de rastreo $0^{5-7}$

Carlos García [ Servicio de Medicina Familiar y Comunitaria del Hospital Italiano de Buenos Aires. carlos.garcia@ hospitalitaliano.org.ar ]

García C. Un porcentaje significativo de los cánceres de pulmón diagnosticados por rastreo con tomografía computada de baja dosis podrían corresponder a sobrediagnósticos. Evid Act Pract Amb. 2015;18(2):36. Abr-Jun. Comentado de: Veronesi, G, et al. Estimating Overdiagnosis in LowDose Computed Tomography Screening for Lung Cancer: A Cohort Study. Ann Intern Med. 2012;157(11):776-784. PMID: 23208167.

\section{Referencias}

1. Kubík AK, y col. Czech Study on Lung Cancer Screening: post-trial follow-up of lung cancer deaths up to year 15 since enrollment. Cancer. 2000;89(11 Suppl):2363-8.

2. Marcus PM, y col. Lung cancer mortality in the Mayo Lung Project: impact of extended follow-up. J Natl Cancer Inst 2000;92(16):1308-16.

3. Welch HG, Black WC. Overdiagnosis in cancer. J Natl Cancer Inst. 2010;102(9):605-13.

4. Patz EF, y col. Overdiagnosis in low-dose computed tomography screening for lung cancer. JAMA Intern Med. 2014:174(2):269-74.

5. Harris RP, y col. The Harms of Screening. JAMA Intern Med. 2014 Feb 1;174(2):281.

6. Sox HC. Better evidence about screening for lung cancer. N Engl J Med. 2011 Aug 4;365(5):455-7.

7. Aberle DR, y col. Reduced lung-cancer mortality with low-dose computed tomographic screening. N Engl J Med. 2011 Aug 4;365(5):395-409. 